LA-UR- $94-3413$

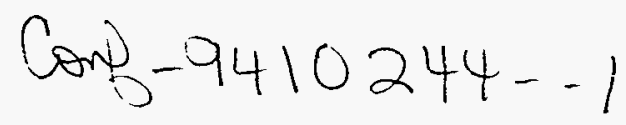

Title: CHARACTERIZATION OF A TEXTURE GRADIENT IN TANTALUM PLATE

Author(s): $\quad$ Stuart I. Wright, MST-6

George T. Gray III, MST-6

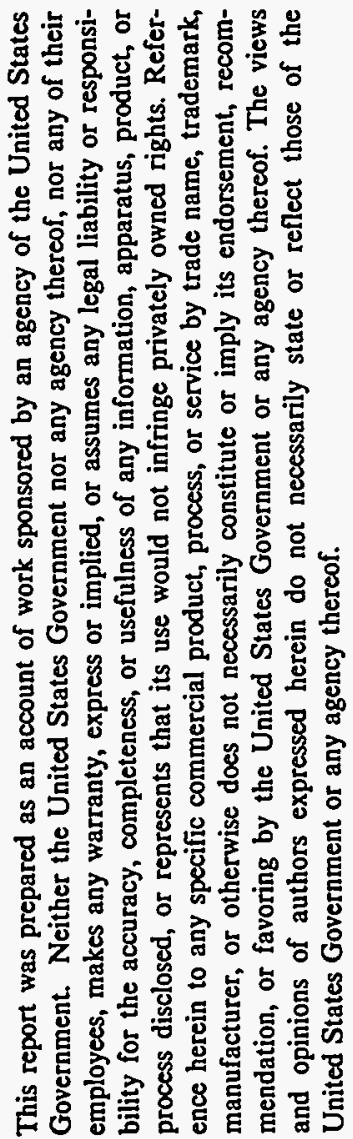

Submitted to:

Third International Symposium on Structural and Functional

Gradient Materials

October 10-12, 1994

Swiss Federal Institute of Technology

Lausanne, Switzerland

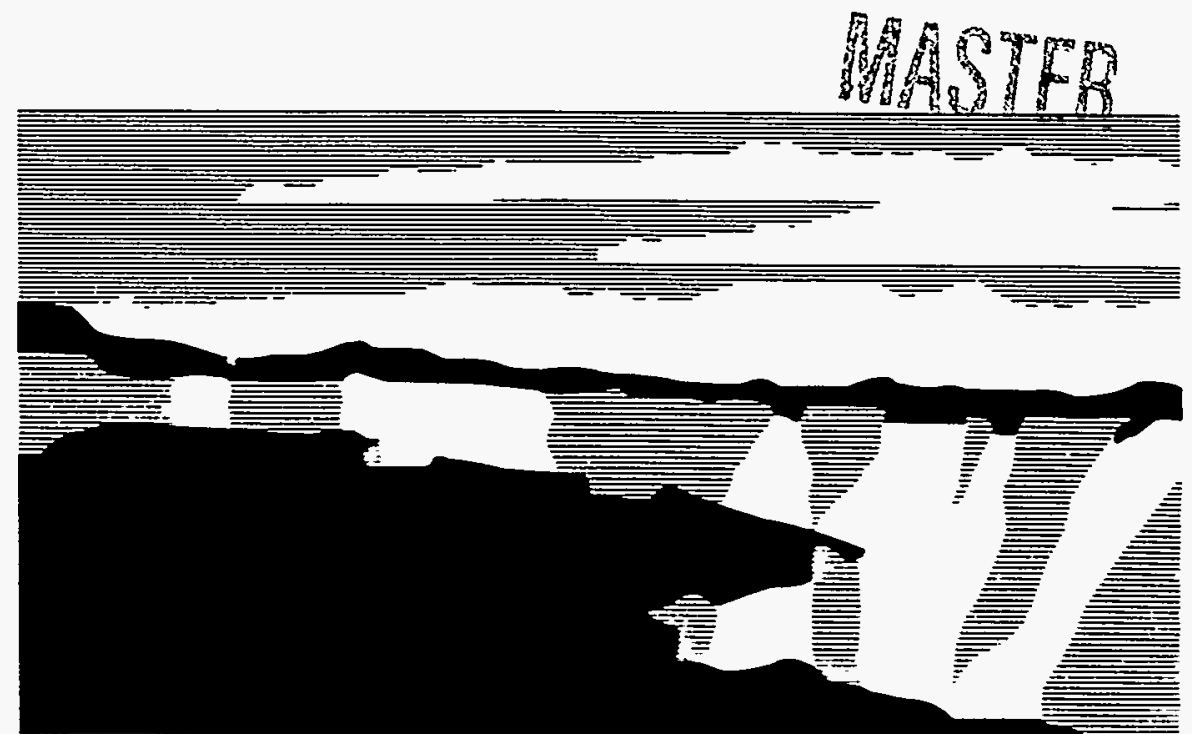

Los Alamos National Laboratory, an attirmative action/equal opportunity employer, is operated by the University of California for the U.S. Department of Energy under contract W-7405-ENG-36. By acceptance of this article, the publisher recognizes that the U.S. Government retains a nonexclusive, royalty-free license to publish or reproduce the published form of this contribution, or to allow others to do so, for U.S. Government purposes. The Los Alamos National Laboratory requests that the publisher identity this article as work performed under the auspices of the U.S. Department of Energy. 


\section{DISCLAIMER}

Portions of this document may be illegible in electronic image products. Images are produced from the best available original document. 


\title{
CHARACTERIZATION OF A TEXTURE GRADIENT IN TANTALUM PLATE
}

\author{
Stuart I. Wright and George T. Gray III \\ Staff Members at Los Alamos National Laboratory, Los Alamos, NM 87545 U. S. A.
}

\section{INTRODUCTION}

Clark et al. [1] have shown that significant texture gradients can be produced in rolled tantalum plate and that the strength of the gradient is dependent on the processing path. Texture gradients are often ignored because they are time consuming to characterize and add significant complexity to materials modeling. The variation in texture through the thickness of rolled materials is most commonly measured by sectioning samples to different depths through the thickness of the plate and then measuring the texture from these section planes by $X$-ray diffraction. A new technique based on automatic indexing of electron backscatter diffraction patterns in the scanning electron microscope $[2,3]$ enables spatially specific orientations to be measured in a practical manner. This technique allows spatial variations in texture to be measured directly enabling gradients in texture to be investigated in more detail than previously possible. This data can be used directly in coupled finite-element/polycrystai-plasticity models to simulate the effects of variations in texture on the plastic behavior of polycrystals.

This work examines the variation in texture through the thickness of a tantalum plate and its resultant effect on the compressive deformation of samples prepared from the plate. The characterization of the texture gradient using the automatic point-by-point measurement technique mentioned above is described in detail. The effect of the gradient on the plastic response of through-thickness compression tests is also discussed. 


\section{EXPERIMENTAL DETAILS}

\subsection{Compression Testing}

Cylindrical compression samples ( $5 \mathrm{~mm}$ diameter, $6.2 \mathrm{~mm}$ length) were electrodischarge machined from rolled and annealed vacuum arc-remelted commercially pure tantalum plate. Specimens were prepared with center axes normal to the principal planes of the plate. The samples were compressed at strain rates of $0.001 \mathrm{~s}^{-1}$ at room temperature.

\subsection{Local Texture Measurements}

The texture was measured on a through-thickness section plane of the undeformed compression specimen and on a through-thickness section plane of a deformed compression specimen. The texture was measured using a fully automated single orientation measurement technique. The samples were mounted in the scanning electron microscope on computer-controlled stages. The electron beam was collimated and focused on the surface of the sample so as to produce electron backscatter diffraction patterns. These patterns were then transmitted to the computer via a high-gain video camera. The computer automatically indexed the patterns to obtain the crystallographic orientation of the diffracting volume in the sample. The stages were translated so as to collect orientation measurements on a prescribed array of locations on the sample. Measurements were made on regular hexagonal grids with $20 \mu \mathrm{m}$ spacing between points (the average grain size was approximately $24 \mu \mathrm{m}) .11,716$ orientations were measured on a $4 \mathrm{~mm} \times 1 \mathrm{~mm}$ grid on the undeformed sample and 11,658 measurements were made on a $2 \mathrm{~mm} \mathrm{x}$ $2 \mathrm{~mm}$ grid on the deformed sample. The locations of these grids on the specimens are shown schematically in figure 1 . In addition to recording the location and orientation at each point in the measurement array the computer also records a parameter describing the quality of the associated diffraction pattern.

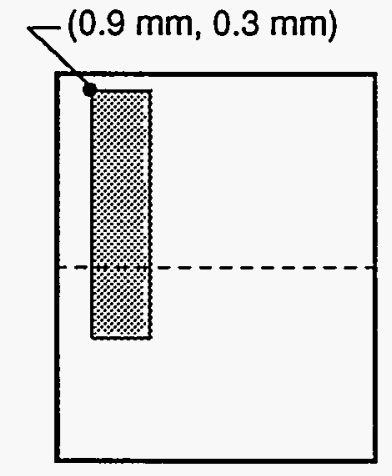

Undeformed

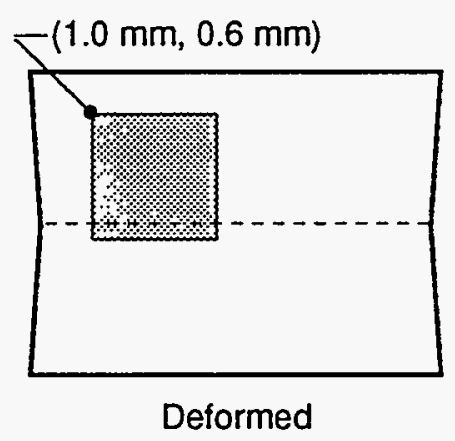

Fig. 1 Schematic of regions on which orientations were measured. 


\section{RESULTS}

\subsection{Compression Testing}

Instead of remaining cylindrical or barreling, the through-thickness compression samples were hourglass shaped after deformation as shown in figure 2. Repeated tests using a variety of lubricants exhibited the same plastic response.

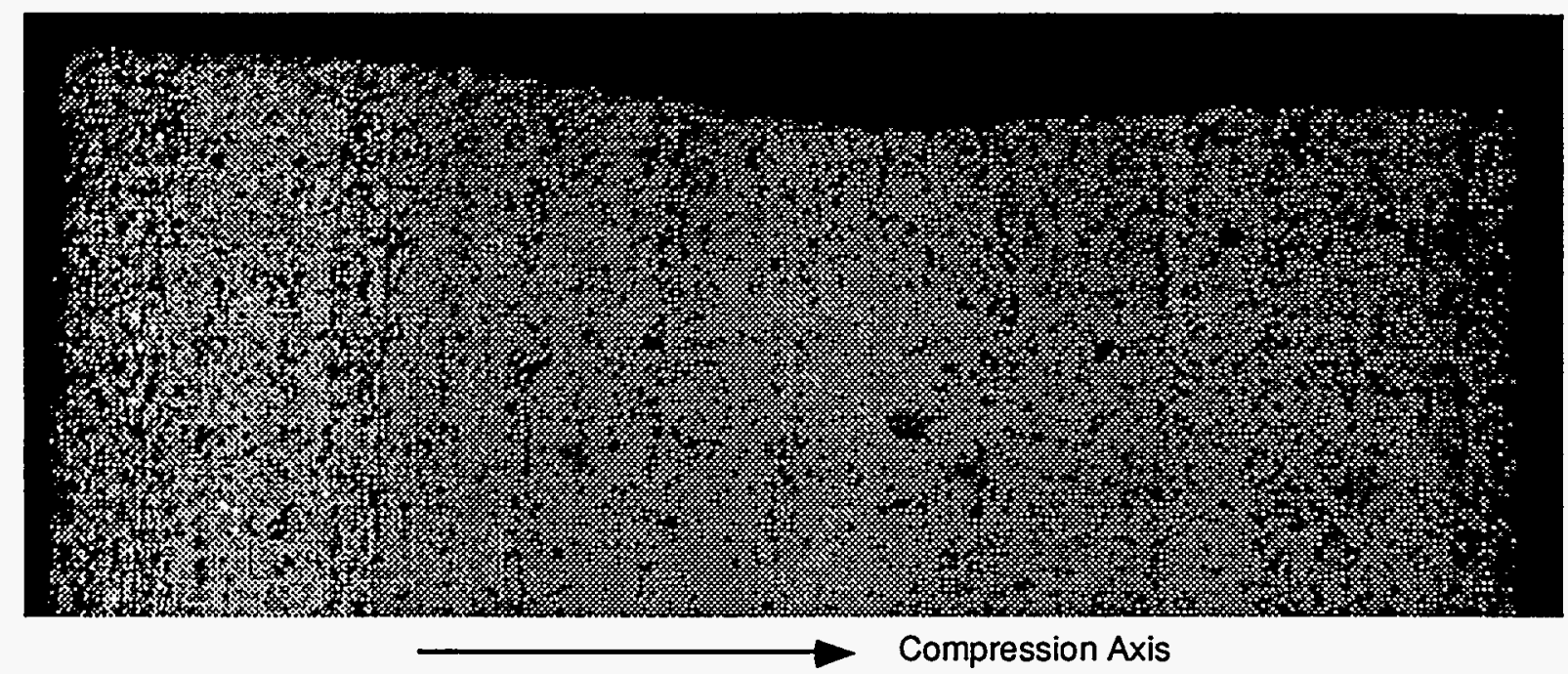

Fig. 2 Section of deformed through-thickness compression sample showing one side of the hourglass profile

\subsection{Local Texture Measurements}

Texture gradients were observed in both the undeformed and deformed samples. However, the gradient was considerably stronger in the undeformed sample. Grains near the plate surface tended to have (001) crystal directions normal to the surface of the plate; whereas, grains near the centerline tended towards having (111) crystal directions normal to the plate surface as can be observed in figure 3 . In addition to the texture gradient, a spatial self-correlation in orientation of the (001) and (111) crystal directions resulting in bands of similarly oriented grains was observed in the texture measurements. 


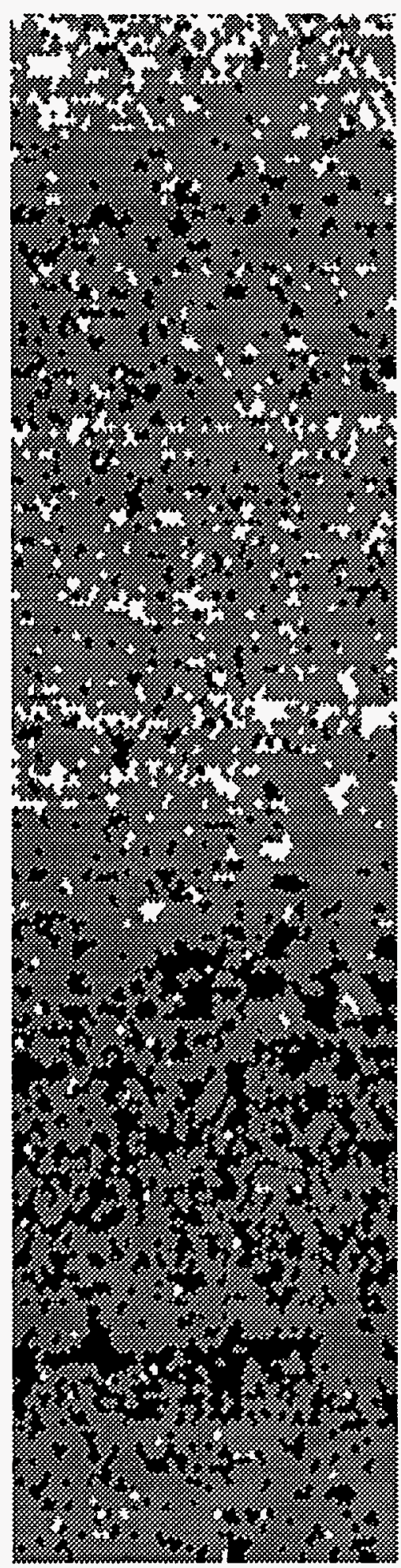

Undeformed Sample
Orientation measurements with (100) crystal axes within $15^{\circ}$ of normal to the plate surface

Orientation measurements with (111) crystal axes within $15^{\circ}$ of normal to the plate surface

Orientation measurements with neither (111) nor (100) crystal axes within $15^{\circ}$ of normal to the plate surface
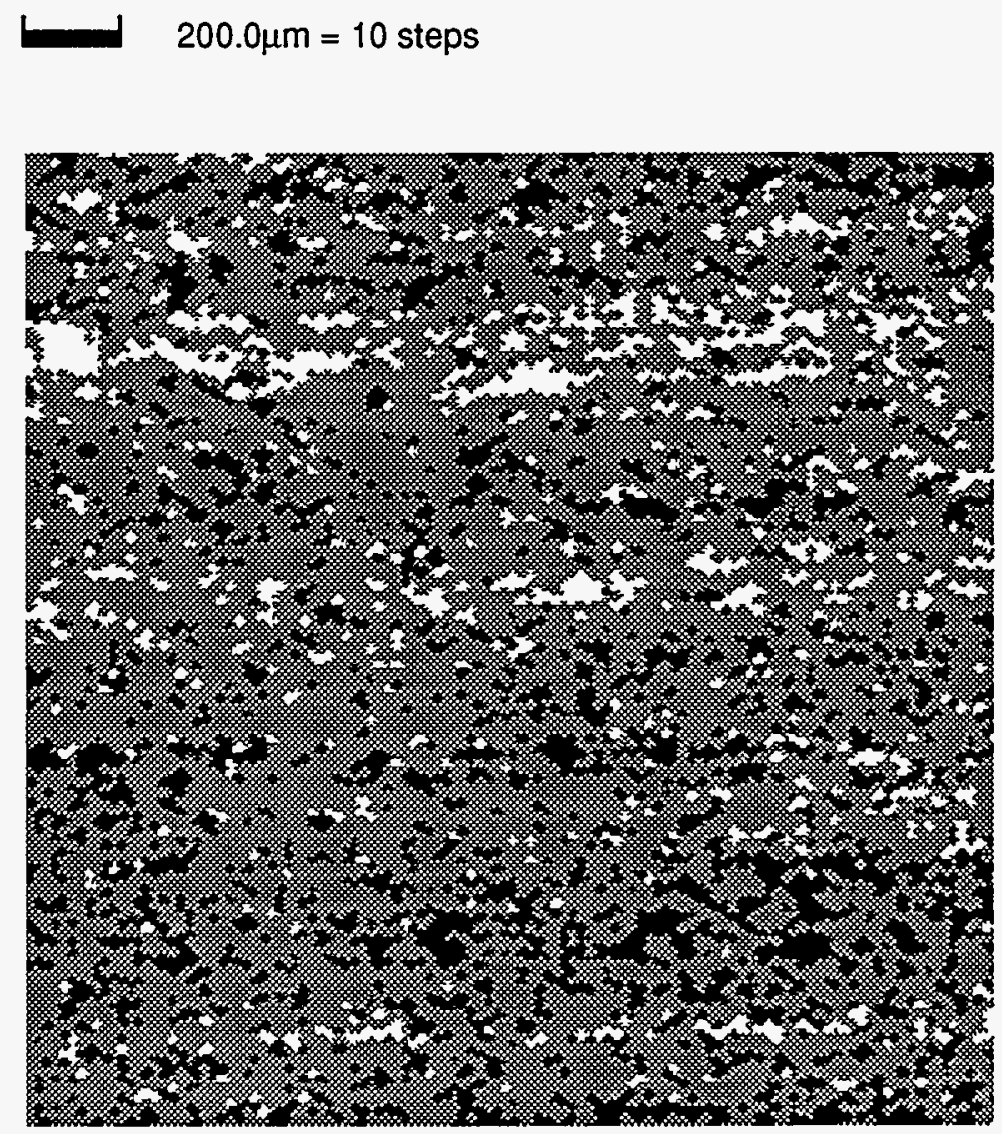

Deformed Sample

Fig. 3 Orientation maps of deformed and undeformed through-thickness specimens. 


\section{DISCUSSION}

As noted earlier, in-plane banding can be observed in the orientation measurements on both the undeformed and deformed samples. Banding can also be observed in the optical micrograph. The bands in the micrograph appear to be formed by grains of similar size. This suggests that there may be a correlation between grain size and grain orientation. No correlation was found, however, since the orientation measurements were taken on a scale very similar to the grain size the measurements had limited value for investigating possible correlations between grain size and grain orientation as has been investigated in [3].

The hourglass shape of the compressed specimens can be attributed to the texture gradient observed in the undeformed sample. The (100) type grains (meaning grains having (100) directions nearly normal to the plate) at the surface of the plate are in a softer orientation than the (111) type grains at the centerline of the plate. This has been confirmed by simulating the compression of the throughthickness sample as described in two previous works [4, 5].

Since the (111) type grains are already in "hard" orientations, the majority of deformation would be accommodated by the (100) type grains and the randomly oriented grains. The quality of electron diffraction patterns is strongly dependent on the amount of strain in the volume from which diffraction occurs $[6,7]$. Thus, we would expect to see a reduction in the average pattern quality for the different types of orientations. However, as shown in figure 4 no discernible effect is observed. This may mean that the degradation in pattern quality is not sensitive enough to measure or that the metric used to quantify the pattern quality is inadequate. The

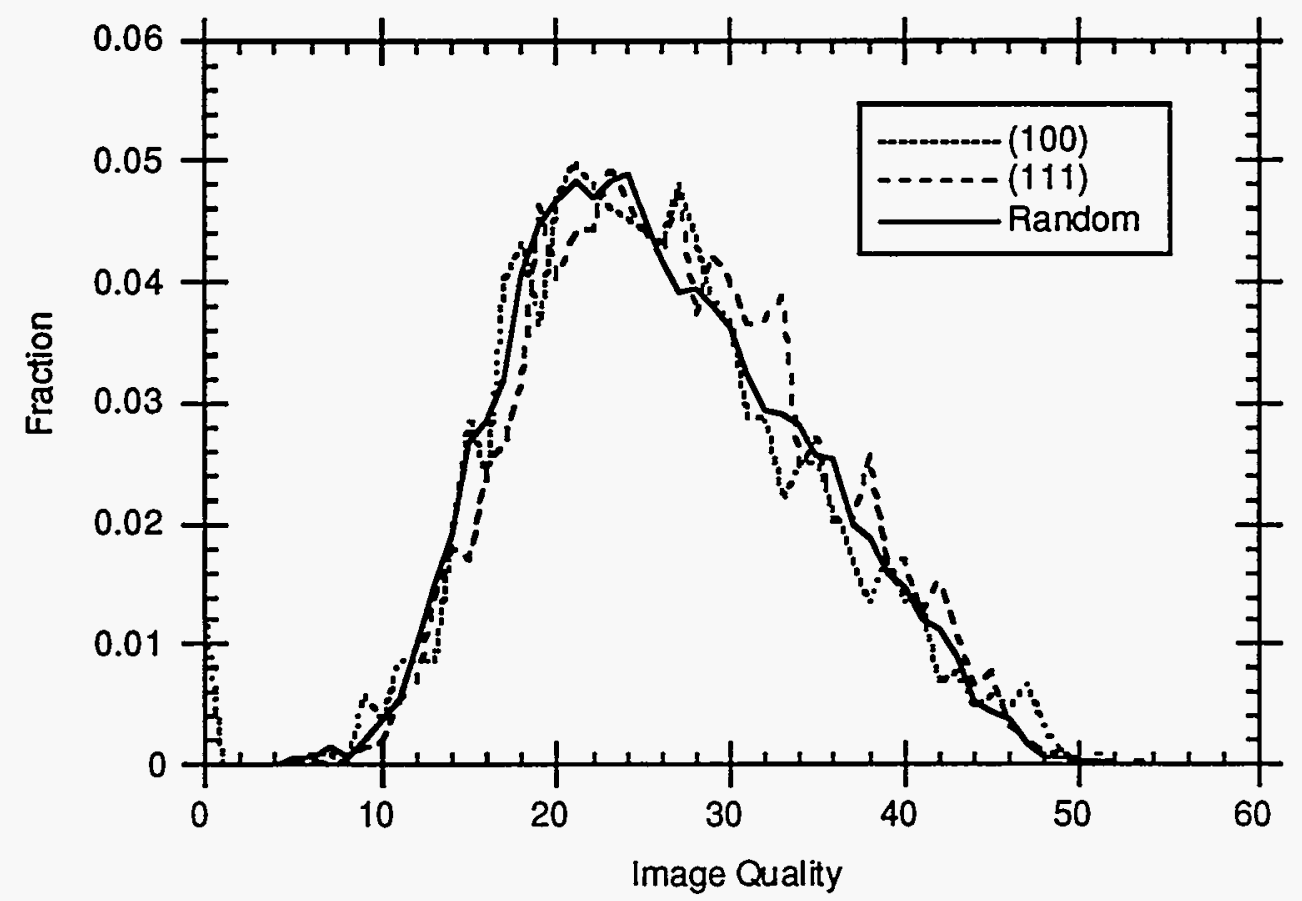

Fig. 4 Distribution of diffraction pattern quality in deformed sample for different orientations. 
absence of any discernible differences in the pattern quality distributions may also be due to the possibility that enough strain was imparted to the sample to camouflage any local differences in pattern quality.

\section{CONCLUSIONS}

The hourglass shape of the deformed through-thickness compression sample can be attributed to a gradient in texture through the thickness of the plate. Grains near the surface of the plate tend to have (100) crystal axes normal to the surface of the plate; whereas, grains nearly the centerline of the plate tend to have (111) axes normal to the surface of the plate. In addition, grains with (100) crystal directions oriented nearly normal to the plate surface were observed to form in-plane bands. The banding was also observed for the (111) type grains.

The automatic technique for making point specific orientation measurements in the scanning electron microscope is well suited for quantitative characterizations of texture and its spatial distribution in polycrystalline materials.. However, further work is needed to determine the capabilities and limitations of the technique for investigating correlations of crystallographic orientation with local strain and grain size.

\section{REFERENCES}

[1] Clark, J. B., Garrett Jr., R. K., Jungling, T. L. and Vandermeer, R. A. "Effect of processing variables on texture and texture gradients in tantalum", Metallurgical Transactions A, vol 22, 1990.

[2] Adams, B. L., Wright, S. I. and Kunze, K., "Orientation imaging: the emergence of a new microscopy", Metallurgical Transactions A, vol 24, 1993.

[3] Wright, S. I., "A review of automated orientation imaging microscopy (OIM)", Journal of Computer-Assisted Microscopy, vol 5, 1993.

[4] Wright, S. I., Gray III, G. T. and Rollett, A. D., "Textural and microstructural gradient effects on the mechanical behavior of a tantalum plate", Metallurgical and Materials Transactions A, vol 25, 1994.

[5] Wright, S. I., Beaudoin, A. J. and Gray III, G. T., "Texture gradient effects in tantalum", Materials Science Forum, vol 157-162, 1994.

[6] Wilkinson, A. J. and Dingley, D. J., "Quantitative deformation studies using electron back scatter patterns", Acta Metallurgica, vol 39, 1991.

[7] Wardle, S. T., Lin, L. S., Cetel, A. and Adams, B. L., "Orientation imaging microscopy: monitoring residual stress profiles in single crystals using an image-quality parameter, IQ", Proceedings of the Fifty-Second Annual Meeting of the Microscopy Society of America, San Francisco Press, San Francisco, 1994. 